\title{
2011-TBD
}

\section{Freezable Radiator Model Correlation Improvements and Fluids Study}

\author{
Sean Lillibridge \\ NASA Johnson Space Center
}

Moses Navarro

Jacobs Engineering (ESCG)

\begin{abstract}
Freezable radiators offer an attractive solution to the issue of thermal control system scalability. As thermal environments change, a freezable radiator will effectively scale the total heat rejection it is capable of as a function of the thermal environment and flow rate through the radiator. Scalable thermal control systems are a critical technology for spacecraft that will endure missions with widely varying thermal requirements. These changing requirements are a result of the space craft's surroundings and because of different thermal rejection requirements during different mission phases.
\end{abstract}

However, freezing and thawing (recovering) a radiator is a process that has historically proven very difficult to predict through modeling, resulting in highly inaccurate predictions of recovery time. To attempt to improve this, tests were conducted in 2009 to determine whether the behavior of a simple stagnating radiator could be predicted or emulated in a Thermal Desktop ${ }^{\mathrm{TM}}$ numerical model. A 50-50 mixture of DowFrost HD and water was used as the working fluid. Efforts to scale this model to a full scale design, as well as efforts to characterize various thermal control fluids at low temperatures are also discussed.

Previous testing and modeling efforts showed that freezable radiators could be operated as intended, and be fairly, if not perfectly predicted by numerical models. This paper documents the improvements made to the numerical model, and outcomes of fluid studies that were determined necessary to go forward with further radiator testing.

\section{Introduction}

T ONG-DURATION missions require spacecraft to endure a wide array of environments. For the thermal $\amalg$ system, this results in distinct challenges in set point temperature management, especially in crewed space flight, where the set point temperature ranges are much more stringent than non-crewed missions. Scenarios comprising high thermal load in a warm environment, as well as low thermal load in a cold environment must be accounted. One way to achieve this sort of variability in the thermal system is to adaptively change the rejection capabilities of the thermal system. As the primary heat rejection technology for a long term lunar mission is a radiator, it makes sense to come up with ways of changing how much heat the radiator is capable of rejecting.

One historically used method for heat rejection variability is through stagnation. This is accomplished by creating a radiator-manifold system that will gradually lose the use of a portion of the radiator tubes in a predictable, repeatable manner. One possible method of accomplishing this task is to use a "C" shaped (manifold inlets and exits are on the same side of the radiator panel, as shown in Fig. 1) manifold design, which results in a flow friction gradient from tube to tube in the radiator, so that the second tube in the radiator has a 
higher resistance to flow than the first, and so on. When the fluid is being run through this parallel radiator setup, flow will be highest in the first tube, second highest in the second tube, and lowest in the last tube.

In action, the mean surface temperature (and the fluid exit temperature) of the radiator is a function of flow rate. The lower the flow rate into the radiator, the colder the radiator will get, as each quanta of fluid has more energy removed from it. With most fluids, viscosity increases with decreased temperature, meaning that as the radiator (and fluid) gets colder, the fluid in the radiator will get more viscous. This effect, however, will not happen equally along all tubes, as the flow rate in each of the tubes (progressing from the first tube to the last) is less than the one before it. Therefore, the fluid in each successive tube will be more viscous than the last.

In fluids like propylene glycol, there is a distinct "elbow" in the temperature-viscosity curve that has the effect of increasing the viscosity quickly through cooling in that temperature regime versus a warmer one (see Fig. 2). This glycol is mixed with water (PGW). If a fluid with this behavior is used in a radiator such as the one described above, stagnation can occur. Once the flow rate is sufficiently low, the last tube will have such a low temperature that the fluid "viscosity elbow" is reached, causing an acute decrease in flow in that tube, resulting in an even lower temperature, and stagnating the tube. This has the effect of bringing the radiator down one tube, effectively eliminating the radiator surface associated with the stagnated tube, as cross conduction through a radiator is typically very poor due to the thin face sheet. The end result of this phenomenon is exactly what is needed for a lunar mission: a radiator capable of varying its heat rejection capability based on a parameter easily controlled by the internal spacecraft thermal control system, namely radiator flow rate. These radiators can even be taken to the point where the farthest tubes freeze and can still recover, albeit slowly. It should be noted that liquid-solid phase change in the radiator flow tubes is the only difference between a freezable radiator and a stagnating radiator. The principles of operation remain the same.

Radiator stagnation is not without its difficulties, however. The stagnation process is very difficult to accurately model, particularly with hybrid fluids such as a propylene-glycol water mix (PGW). Difficulties encountered include conductivity along the fluid when in creep or zero flow conditions, among others. Initial efforts to

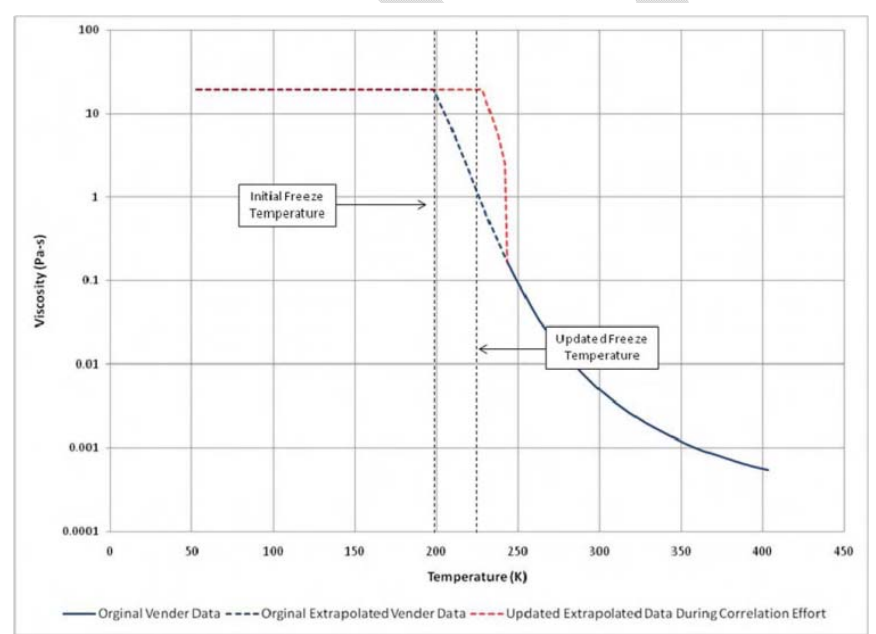
model test data resulted in results representative of Fig. 3 on the next page. As can be seen with those results, recovery still posed a challenge for the freezable radiator model, with temperatures during recovery being modeled as much as $50^{\circ} \mathrm{F}$ off of their empirical results. This shows the clear need for further model development if a freezable radiator is to be used in future spacecraft designs.

Figure 2: Increase in viscosity with decreased temperature. 
Mid-Point between Outlet Manifold and Radiator Mid-Point Tube Temperatures

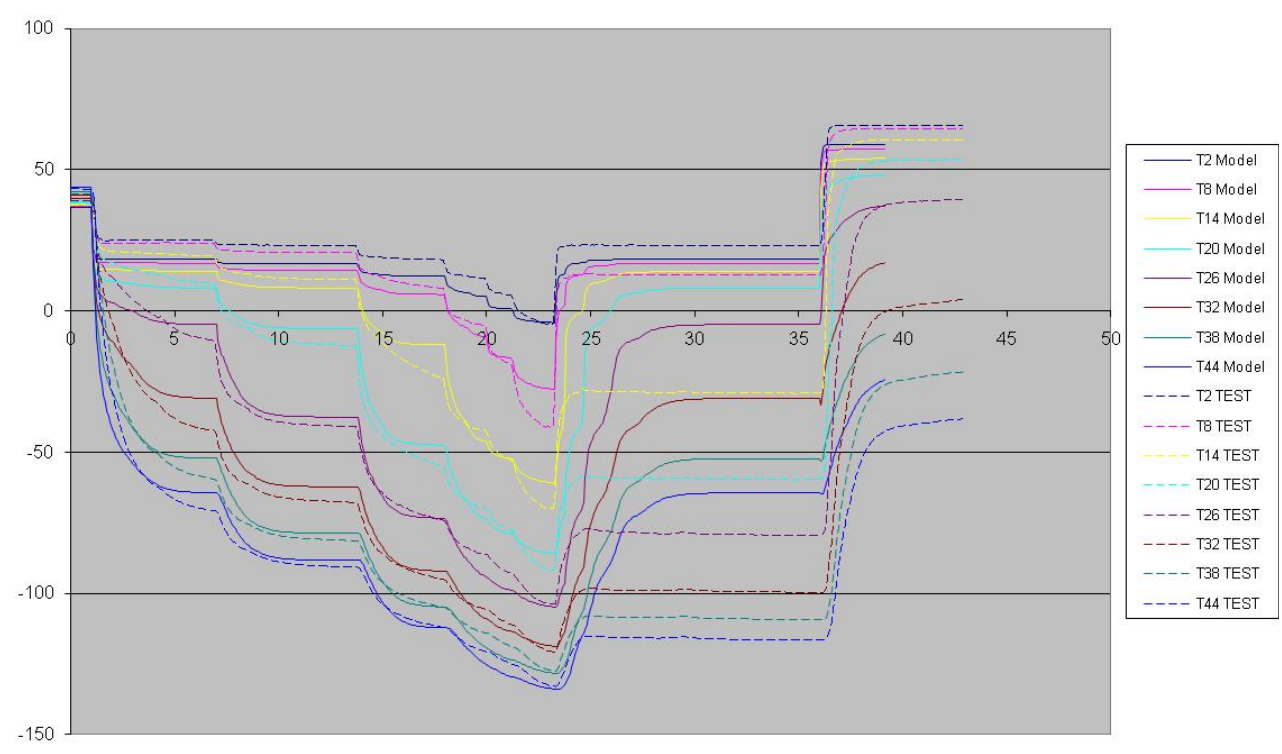

Figure 3: Empirical and modeling results for a long freeze test. Recovery poses a difficulty in model prediction of radiator performance.

\section{Modeling Improvements}

In order to improve the performance of the Thermal Desktop $^{\mathrm{TM}}$ model, a piecewise evaluation was adopted; where each variable's possible errors were minimized serially and checked for their effect on the model solution. This paper will give an overview of methods and how each variable affected the thermal model. For an in-depth explanation of the physics behind the thermal model, please reference Navarro, 2010 in the reference section.

\section{Mass}

Increasing accuracy in the mass distribution of the thermal model was the first variable to be addressed. The most significant mass change made was the addition of the heater mass into the model. The heat trace lines which were used during testing to manually recover the radiator panel from a hard freeze (used only when normal recovery was impossible) had not been previously modeled, and the net reduction caused the model to predict that the radiator would respond too quickly to a change in mass flow rate. Each heater added 160 grams of thermal mass to the tubes, which brought transient response more in line with experimental results. With all eight tubes this added nearly $1.3 \mathrm{~kg}$ to the overall radiator mass.

\section{Heat transport}

Flow paths were calculated using FLUINT short tubes (STube connectors), thus fluid inertia was neglected. Furthermore, minor loss coefficients used in the model for the manifold junctions were eventually removed because they were inaccurate for the Reynolds numbers typically seen during testing (less than 100).

A unique freeze logic was used to model fluid freezing in the tubes for this test, which observes a cooling fluid getting to its freeze temperature, and reduces the flow path diameter. Eventually this reaches a very small diameter, and flow is cut. However, in doing this, the conductive area to the tube wall was also found to decrease, eventually decreasing to zero. This has the effect of inaccurately isolating the fluid slug in the tube from the tube wall, and needed revision. To solve this, an additional variable was set to allow for fluid slug to tube wall thermal conductivity once the fluid flow had been shut off. This allowed the frozen fluid to continue to cool along with the rest of the radiator, resulting in better transient performance as the fluid mass is accounted for as the radiator cools down below freezing temperatures.

\section{Fluid Thermophysical Properties}

The fluid thermophysical properties originally used for the freezable radiator model were known only down to about $-30^{\circ} \mathrm{C}$. As the radiator got significantly colder, thermophysical properties were needed below $-30^{\circ} \mathrm{C}$. This was accomplished by using a curve fit with the known vendor data. Additionally, a spike in specific heat was inserted into the data tables to account for phase change at an assumed freeze temperature of 198.15K. It became increasingly apparent for experimental situations where the radiator experienced temperatures below 227.6K for extended periods of time that the initial fluid model assumptions were unacceptable, and caused 


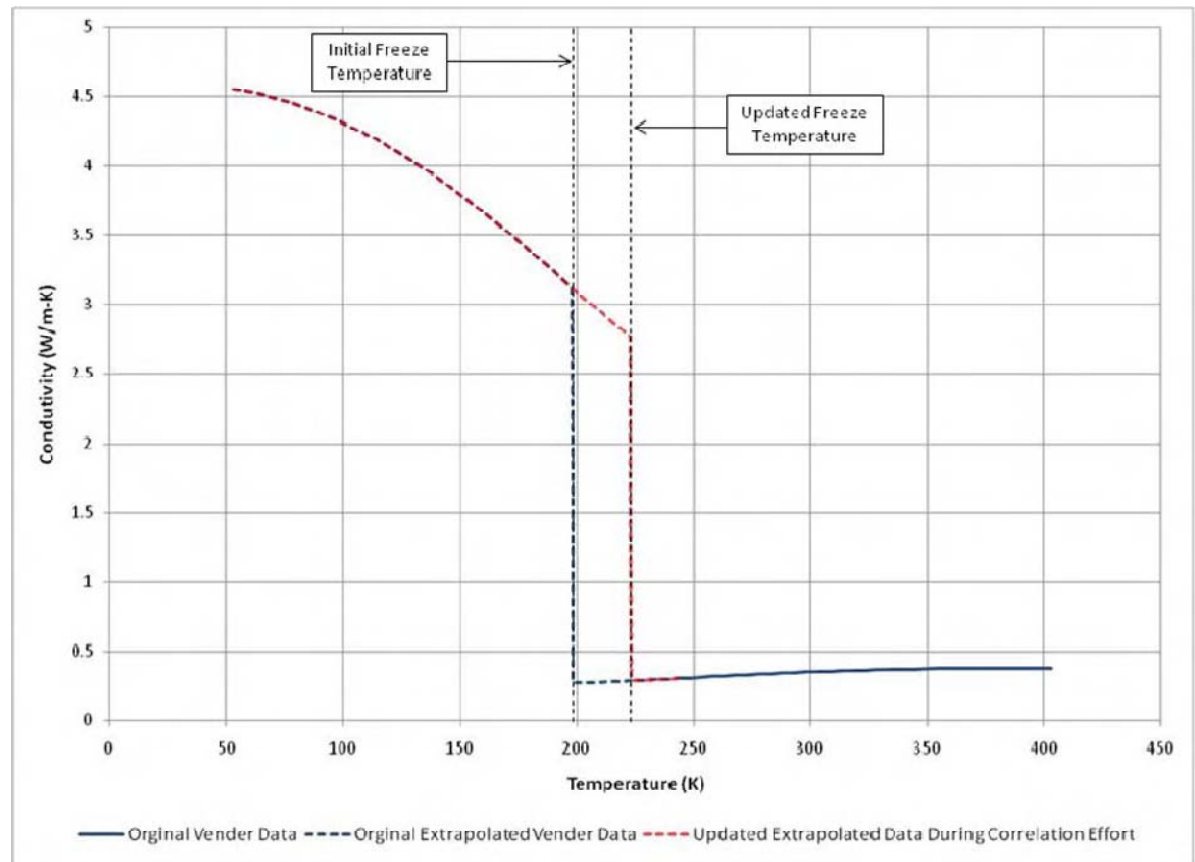

Figure 4: Thermal conductivity of 50/50 PGW, indicating change in freeze temperature. large steady state error. As a result, the fluid thermophysical properties were brought into revision as the modeling effort progressed.

The first major revision came from qualitative testing conducted at JPL, wherein a beaker of 50/50 propylene glycol/water (PGW, by mass) was taken to freezing temperatures and observed for behavior visually. The mixture was seen to freeze closer to $223 \mathrm{~K}$ rather than 198K, resulting in a change in the location of the specific heat spike, and changing the point at which the other thermal properties were transitioned from fluid to solid properties.

\section{Density}

The change in phase change temperature seen in the JPL test resulted in a small change in the assumed density, shown in Fig. 4 above. This was found to have a minor transient improvement in the model, as very small mass improvements were made with regard to the total mass of fluid frozen in the radiator tubes. However, this was not found to have a significant effect on thermal testing.

\section{Thermal Conductivity and Specific Heat}

Like density, thermal conductivity and specific heat had shifts in where they transitioned from liquid to solid properties with the changed freeze temperature, however, these changes to the thermal properties provided only incremental improvements to the ability of the model to predict test results. Shifts to thermal conductivity and specific heat are shown in Figs. 4 and 5, respectively.

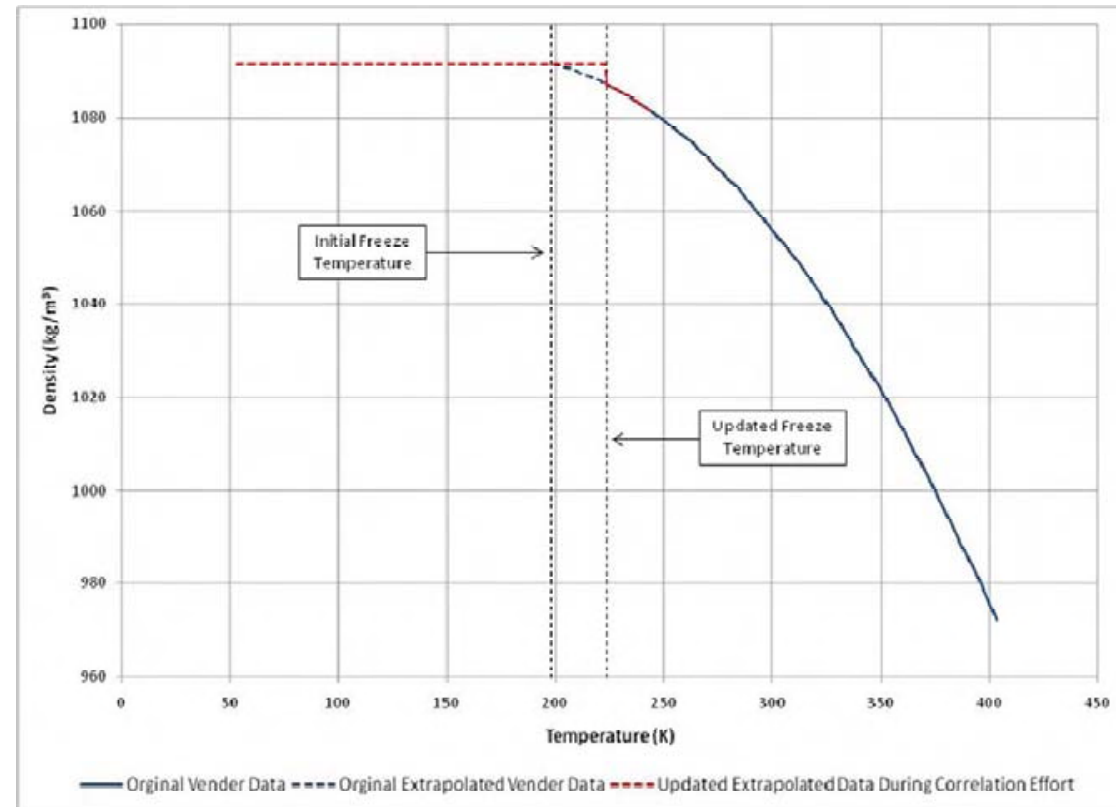

Figure 5: Assumed 50/50 PGW Density, showing revision of phase change temperature. 


\section{Viscosity}

Viscosity had the strongest effect on model performance. The model was found to be strongly responsive to even small changes in viscosity; changes of less than a percent created a recovery temperature swing of more than $50^{\circ} \mathrm{F}$. Initially the curve was updated to follow the freeze temperature as with the other thermophysical properties, but further refinements, as shown in Fig. 2, showed further model improvements. These additional features in the curve, the ramp rate leading up to the freeze viscosity, were solved for by iteratively modifying the property deck and checking for model performance vs. experimental results after each small modification. At this point it became clear that the thermal model would need to rely on more accurate fluid property measurements than had currently been obtained for temperatures below $-30^{\circ} \mathrm{C}$, which will be discussed below.

\section{$\underline{\text { Model Results after changes }}$}

After the iterative customization of the viscosity curve and updates to the other thermophysical properties related to the freeze temperature modification, as well as the other modifications mentioned above, were

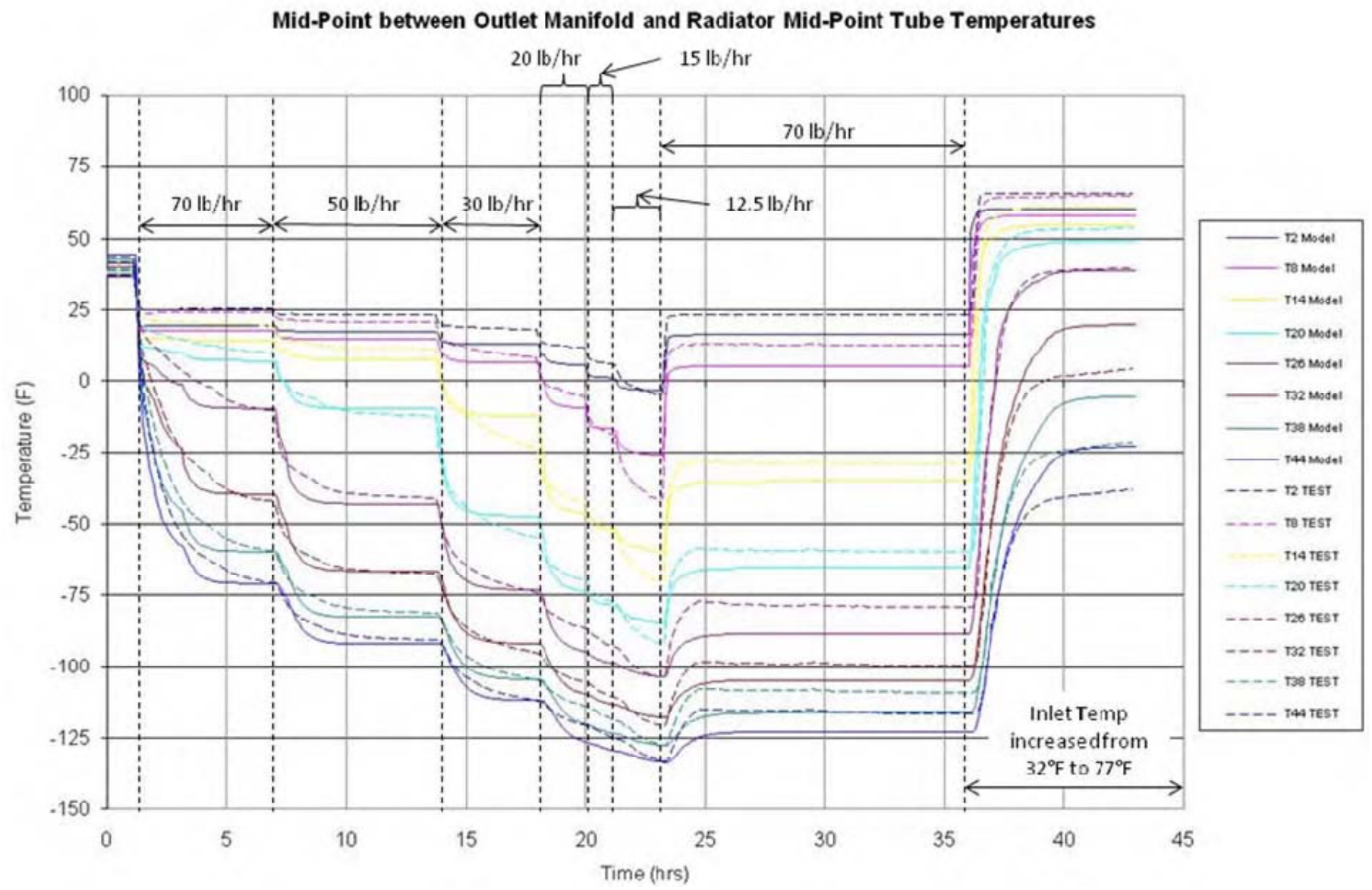

Figure 7: Representative thermal model results, after modifications. 
completed, the model became much better at replicating experimental results. Seen on the next page (Fig. 7) is the same test run as seen in Fig. 3, matched with the results of the modified freezable radiator model. While transient performance is still slightly off, temperatures reach a much closer steady state values during flow rate step-down toward stagnation at the beginning of the test run. Upon recovery (the right half of the graph in Fig. 7) steady state temperatures are much improved, seeing typical deltas of $10^{\circ} \mathrm{F}$ or less between model and experiment.

While it is an accomplishment to have such improved agreement between the test article and numerical model, this accomplishment was dependent upon the alteration of thermophysical properties of the working fluid. In order to have confidence in the models results, the assumed thermophysical properties must be confirmed by fluids testing.

\section{Fluids Study}

Previous qualitative testing done at JPL indicated an erratic freeze pattern in 50/50 PGW [figure to be added]. Because of this there was motivation to find a thermal control fluid that would behave more predictably as it froze. After gathering information on a battery of fluids available and ruling out fluids not suitable for humanrated vehicles due to toxicity or flammability, the following fluids were selected (Table 1).

Table 1: Fluids subjected to quantitative analysis.

\begin{tabular}{|c|c|c|c|}
\hline Name & Composition & Manufacturer & $\begin{array}{c}\text { Reported Freeze/Glass } \\
\text { Transition Temperature }\end{array}$ \\
\hline Amsoil ANT & $\begin{array}{c}50 / 50 \text { mixture of corrosion-inhibited } \\
\text { propylene glycol and deionized water }\end{array}$ & Amsoil & $\sim-30^{\circ} \mathrm{C}$ \\
\hline Q-Therm SZ2 & Proprietary water-based mixture & Mainstream & $-25^{\circ} \mathrm{C}$ \\
\hline MultiTherm WB-58 & Proprietary water-based mixture & MultiTherm & $-58^{\circ} \mathrm{C}$ \\
\hline Galden HT-170 & Perflourinated polyether & Solvay Solexis & $\sim-100^{\circ} \mathrm{C}$ \\
\hline
\end{tabular}

Because this paper is primarily about model correlation of our previous testing, thermophysical results of the three non-propylene glycol fluids can be found in the appendix for reference.

Density, specific heat, thermal conductivity, and viscosity were tested at various laboratories capable of fluids testing in the $-30^{\circ} \mathrm{C}$ to $-100^{\circ} \mathrm{C}$ temperature range. Table 2 shows the laboratories utilized for testing, as well as the testing methods for each test. 
Table 2: Laboratories used for thermophysical testing

\begin{tabular}{|c|c|c|c|c|}
\hline Laboratory & $\begin{array}{c}\text { Specific } \\
\text { Density } \\
\text { (pyknometer) }\end{array}$ & $\begin{array}{c}\text { Thermal } \\
\text { (Differential } \\
\text { scanning } \\
\text { calorimeter) }\end{array}$ & $\begin{array}{c}\text { Conductivity } \\
\text { (heated } \\
\text { probe) }\end{array}$ & $\begin{array}{c}\text { Viscosity } \\
\text { (rheometer) }\end{array}$ \\
\hline $\begin{array}{c}\text { Thermophysical Properties Research } \\
\text { Laboratory, Inc. (West Lafayette, IN) }\end{array}$ & $\mathbf{X}$ & $\mathbf{X}$ & $\mathbf{X}$ & $\mathbf{X}$ \\
\hline Polymer Solutions (Blacksburg, VA) & & & & $\mathbf{X}$ \\
\hline TA Instruments (New Castle, DE) & & & & \\
\hline
\end{tabular}

Density, specific heat, and thermal conductivity were found to have minimal effects on the overall performance of the thermal model, making slight improvements to the transient responses only. Results of these tests can also be seen in the Appendix.

Viscosity testing for Amsoil ANT showed some variation, however the results, seen in Fig. 8, are encouraging. Both testing laboratories confirmed the general behavior assumed by the thermal model. While the values are not identical, this can be accounted for by the variability in the mixtures tested as well as the unpredictability of PGW's freezing. It should also be noted that while Amsoil ANT was tested in a 50/50 mixture of DI water by mass and is approximately the same volume of propylene glycol as Dowfrost HD, Dowfrost HD was used in solution for the testing being used to evaluate the radiator model.

\section{Discussion}

Ultimately the most influential variable thermophysical fluid property on the model was viscosity. The viscosity curve found iteratively that allowed the fluid model to operate was proven to be indicative of the actual behavior of 50/50 PGW solutions at low temperatures, adding confidence to the accuracy of the model and its ability to be used to predict the performance of freezable radiators in thermal control systems.

\section{Conclusions}

The thermal model developed to predict the performance of a freezable radiator has shown that it can reproduce the behavior of two thermal models using a thermal environment that is generally scalable to other test articles using a 50/50 PGW solution.

Going forward, the model will be tested against MultiTherm WB-58

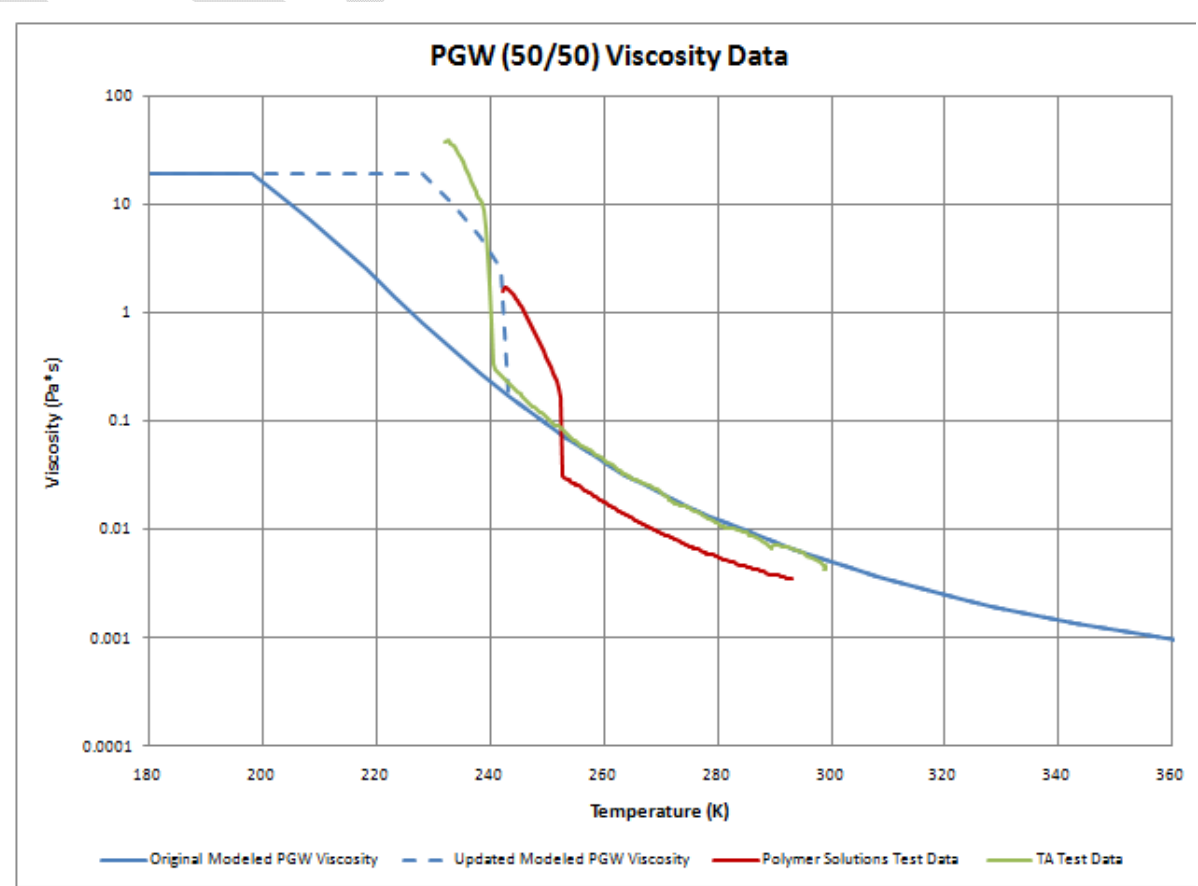

Figure 8: Viscosity of Amsoil ANT 50/50 solution, experimental, modeled, and vendor data. 
in a 6 tube radiator which has been designed to be a subsection of a full scale freezable radiator design. This radiator will also be tested with Amsoil ANT to test for repeatability in the model with different geometries. Should the model prove capable of predicting performance of this new freezable radiator, we can with some confidence proceed to a full scale radiator design.

\section{References}

Lillibridge, S.T., Navarro, M., Cognata, T., \& Guinn, J. (2010). Freezable radiator testing. Proceedings of the International conference on environmental systems American Institute of Aeronautics and Astronautics.

Lillibridge, S. . NASA, EC-2. (2011). Quantitative analysis of fluid thermophysical properties at low temperatures. Houston, TX: CTSD.

Navarro, M. Engineering and Science Contract Group, EC2. (2010). Assessment of a freezable radiator for variable heat rejection (ESCG-4470-10-TEAN-DOC-0067). Houston, TX: Jacobs Engineering. 


\section{Appendix}

Density

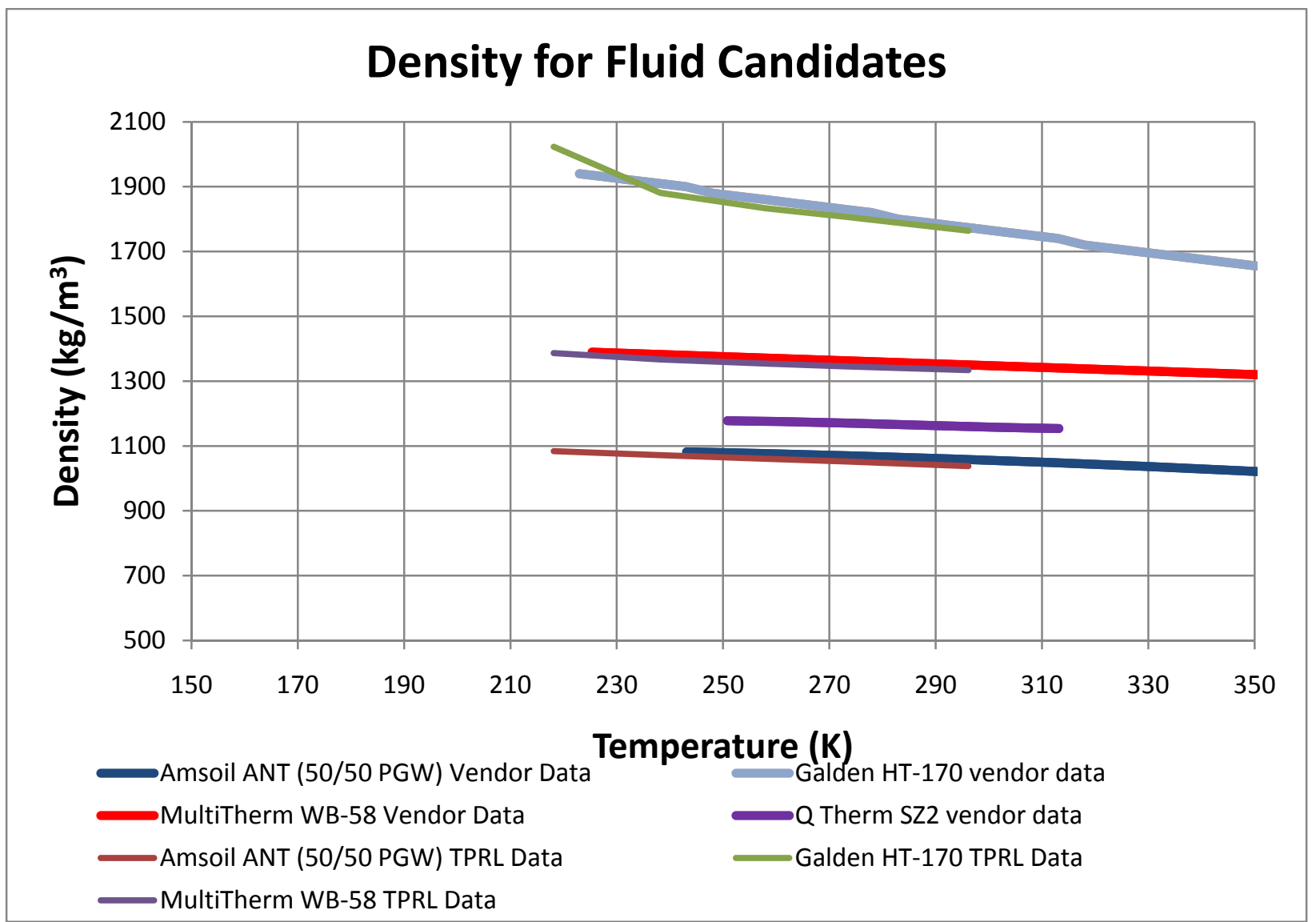

Figure 9: Density of fluid candidates studied, including vendor and test data. 
Specific Heat - Note that MEL data is experimental and is added here only for completeness.

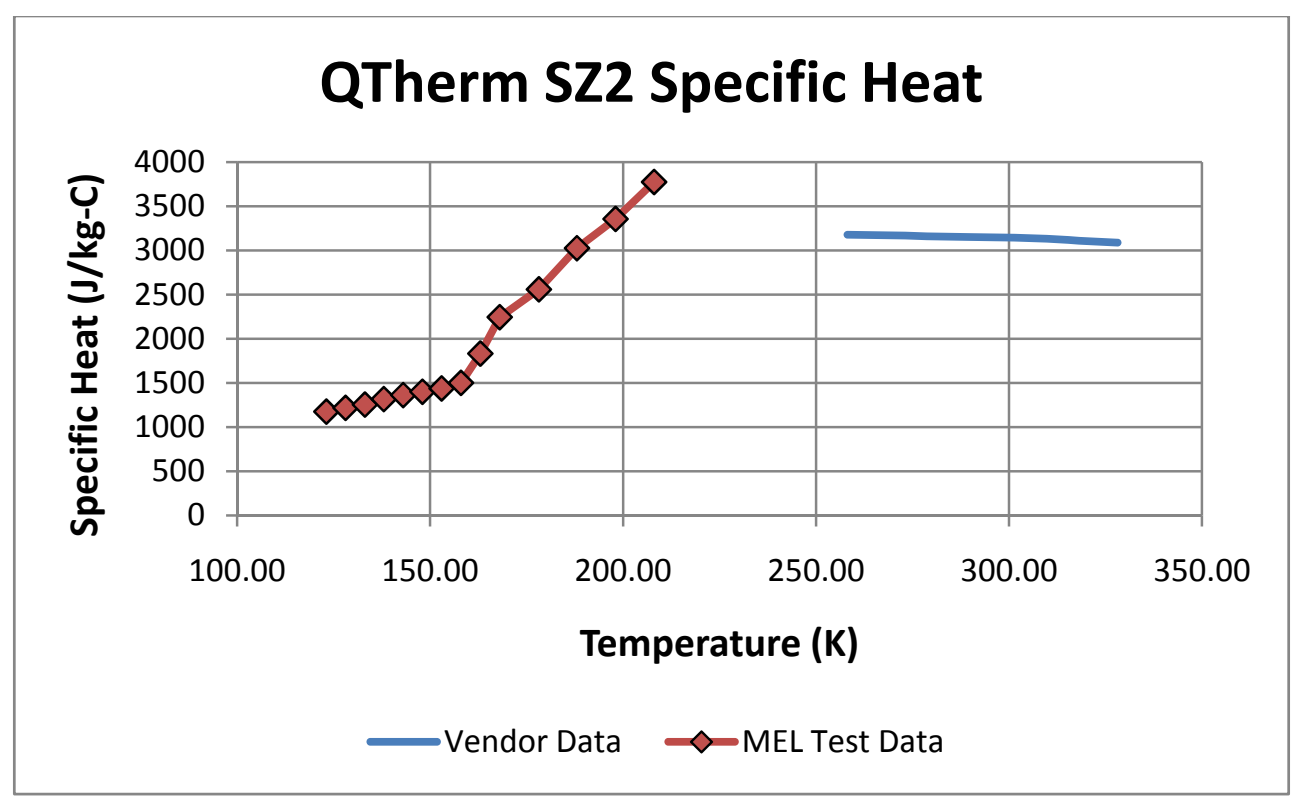

Figure 10: QTherm SZ2 Specific Heat

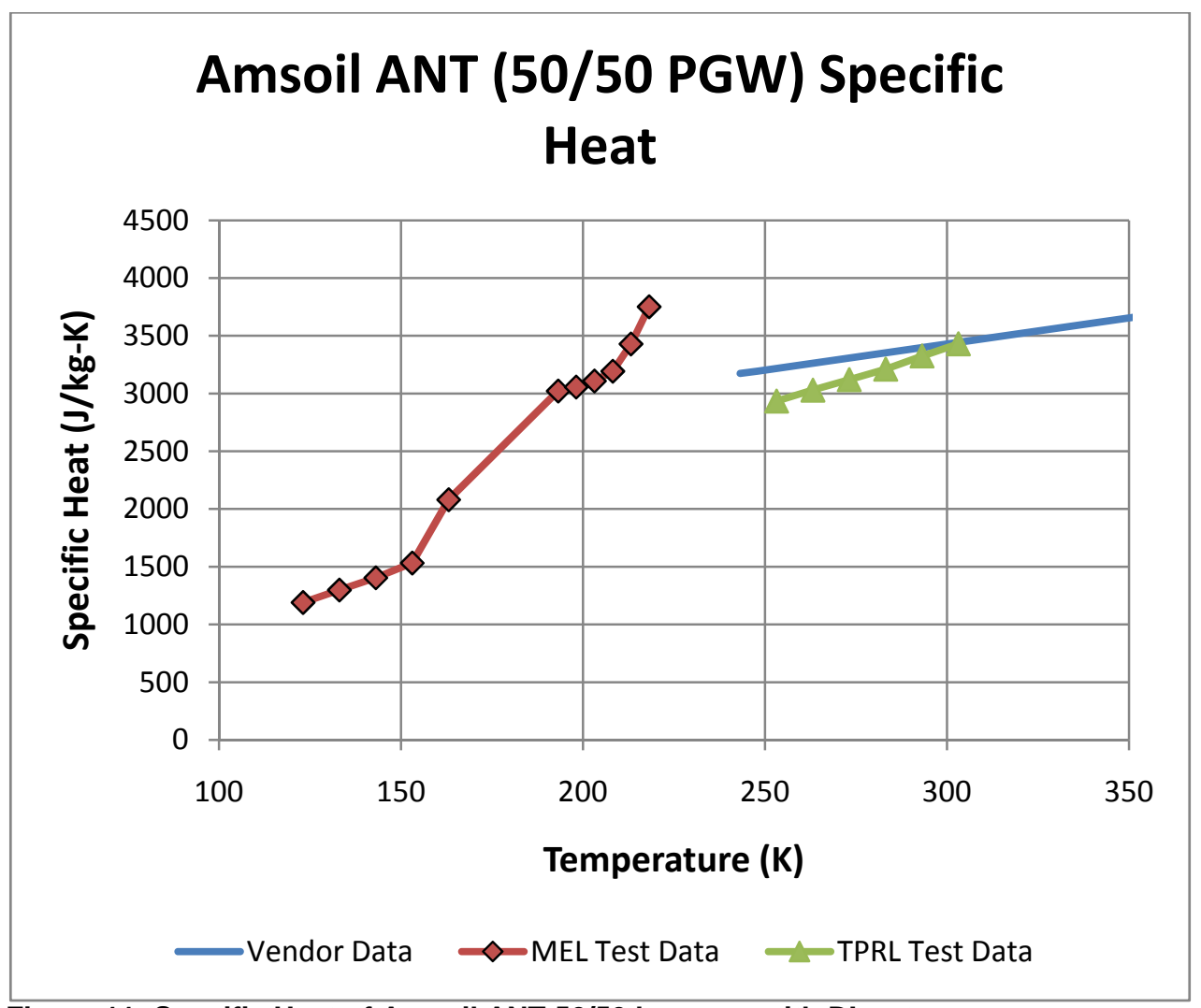

Figure 11: Specific Heat of Amsoil ANT 50/50 by mass with DI water 


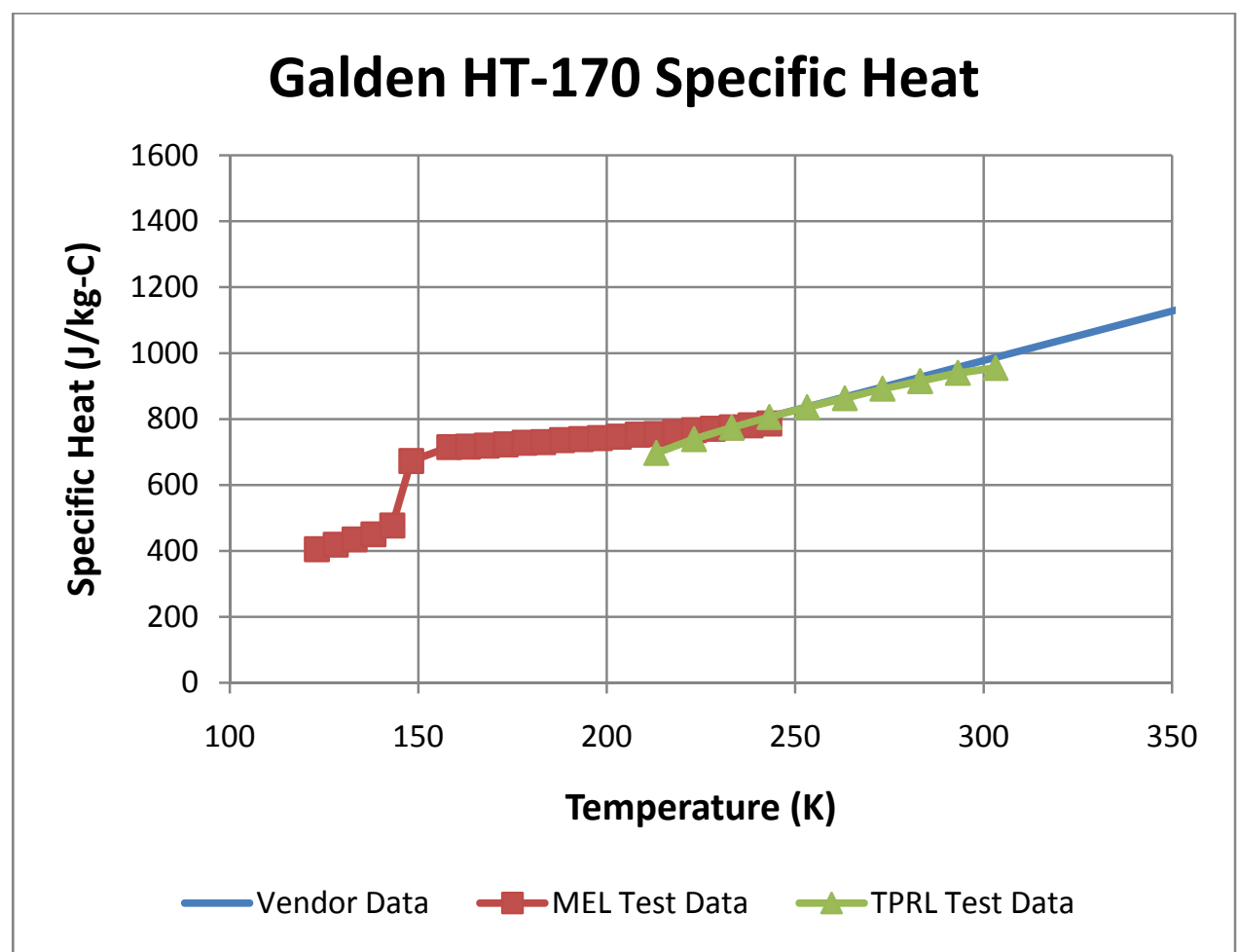

Figure 12: Specific Heat of Galden HT-170

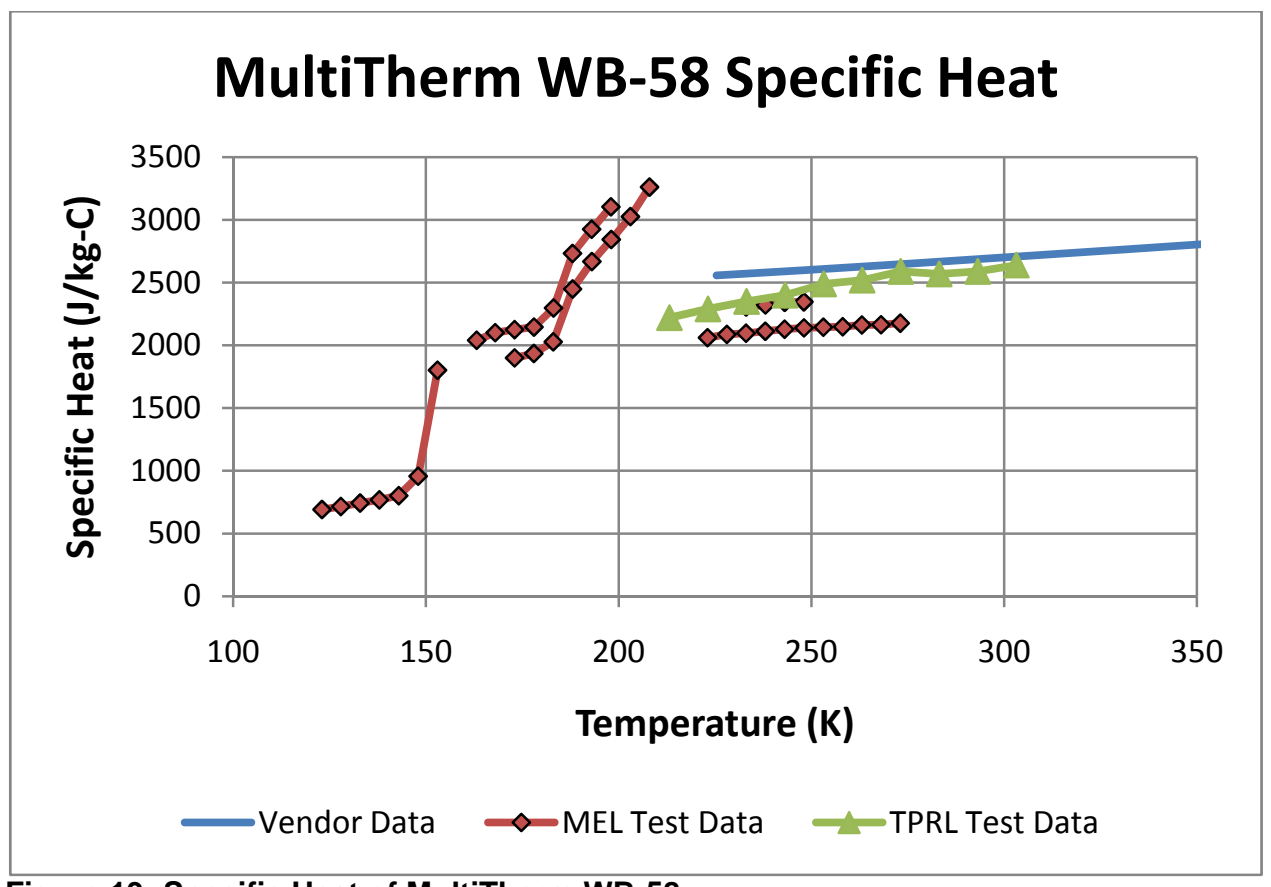

Figure 13: Specific Heat of MultiTherm WB-58 


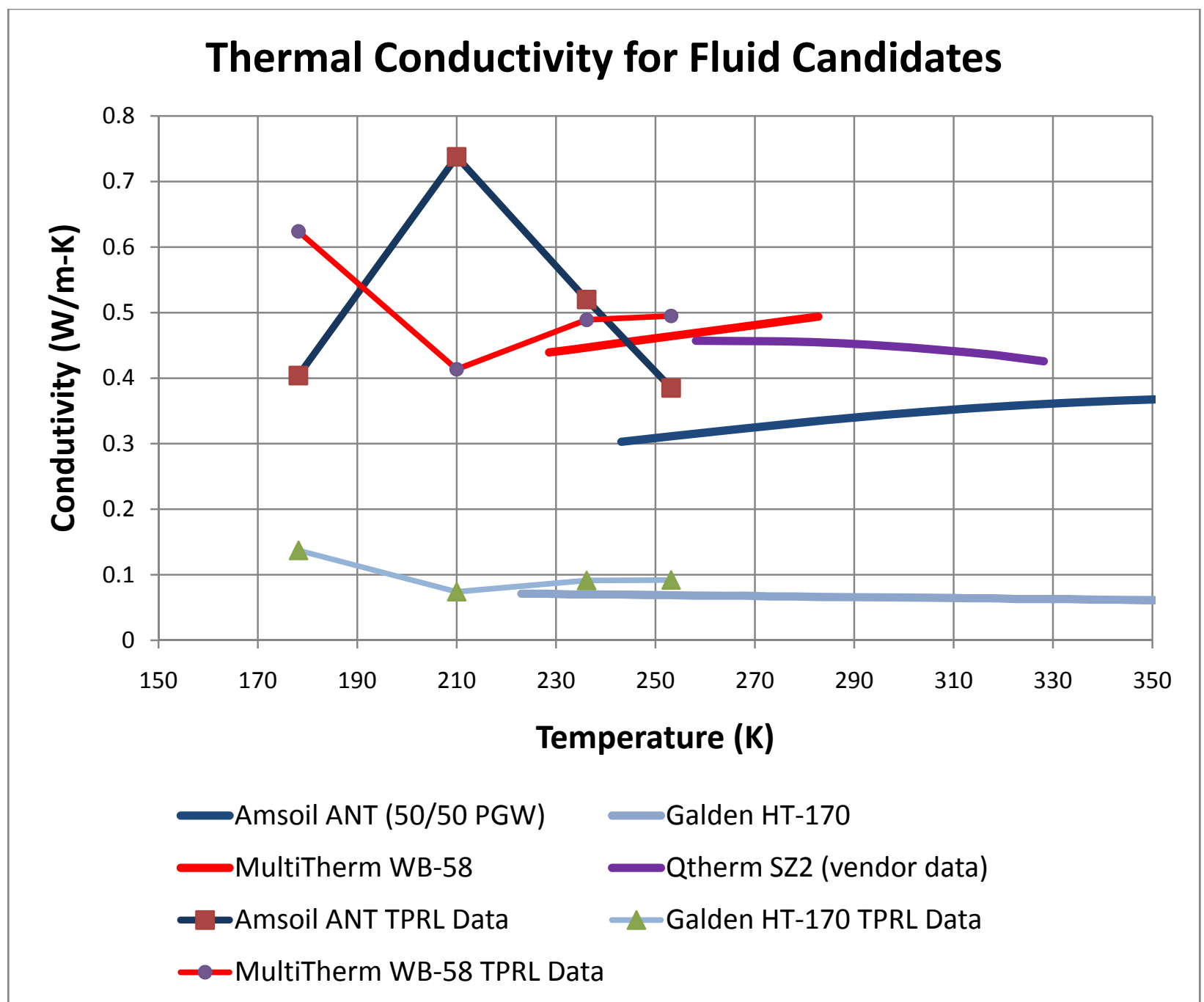

Figure 14: Thermal Conductivity of fluid candidates. Solid lines represent vendor data, lines with data markers represent TPRL test data. 
Viscosity

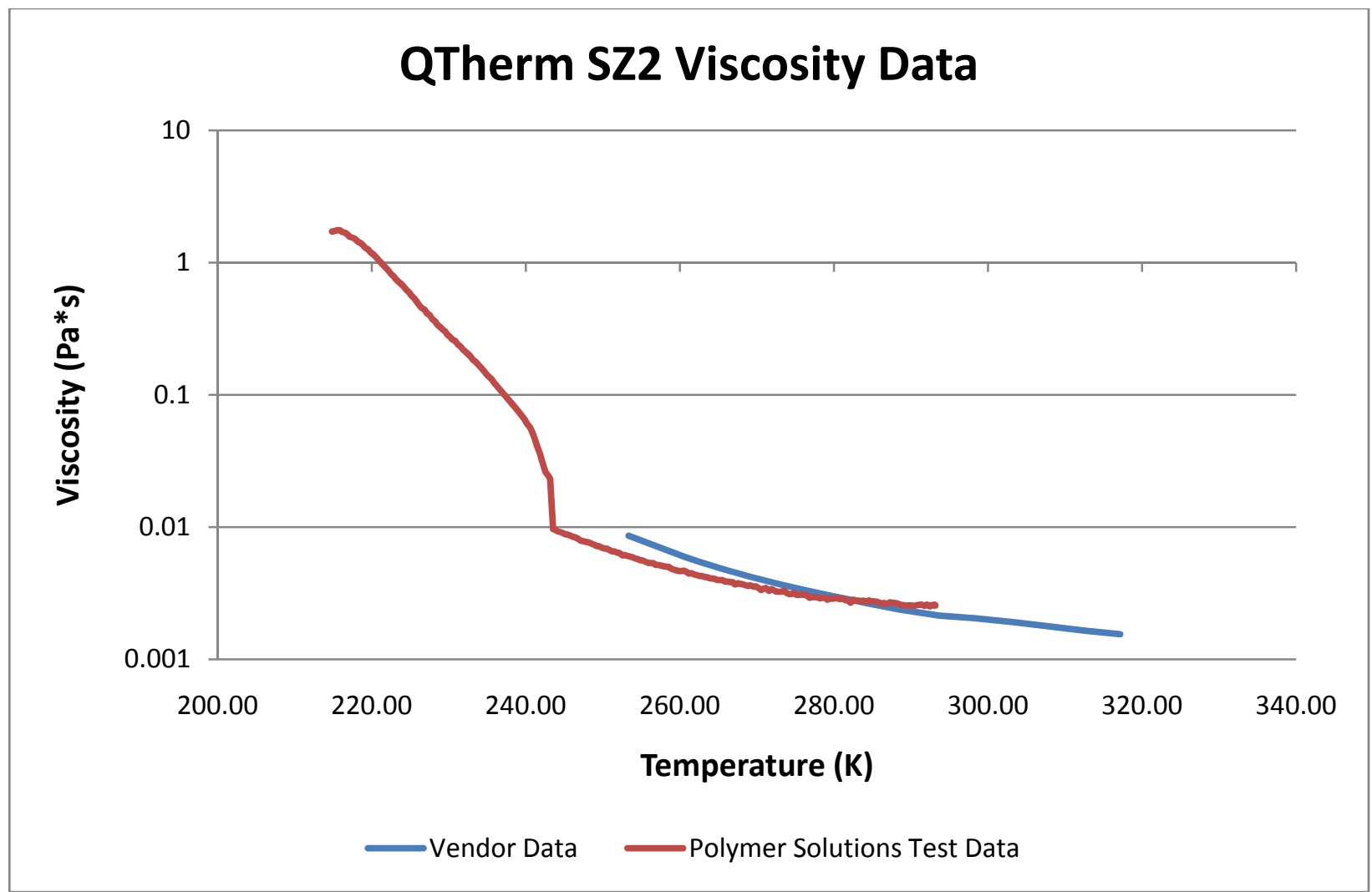

Figure 15: Viscosity profile of QTherm SZ2 with respect to temperature.

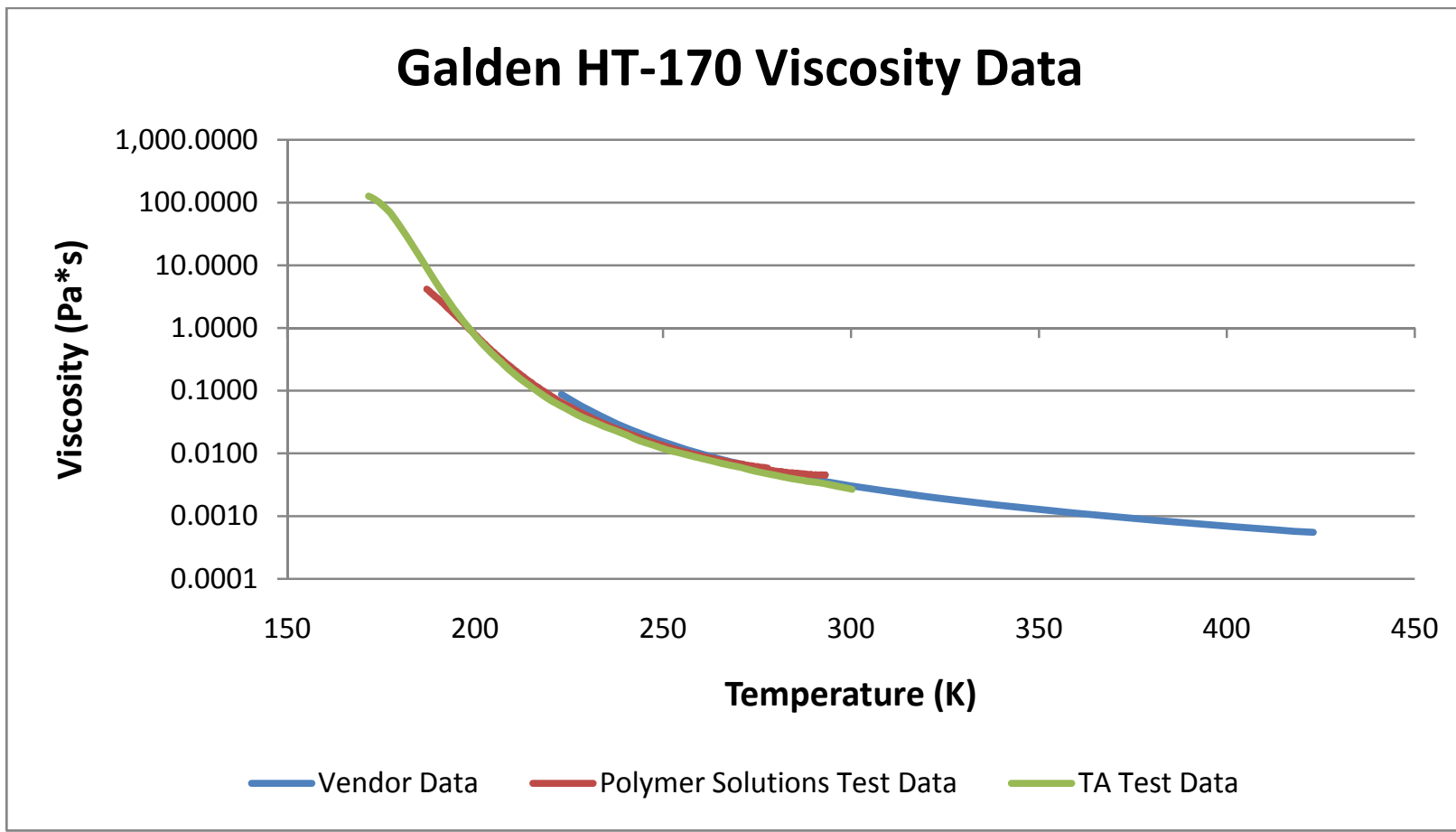

Figure 16: Galden HT-170 Viscosity test data 


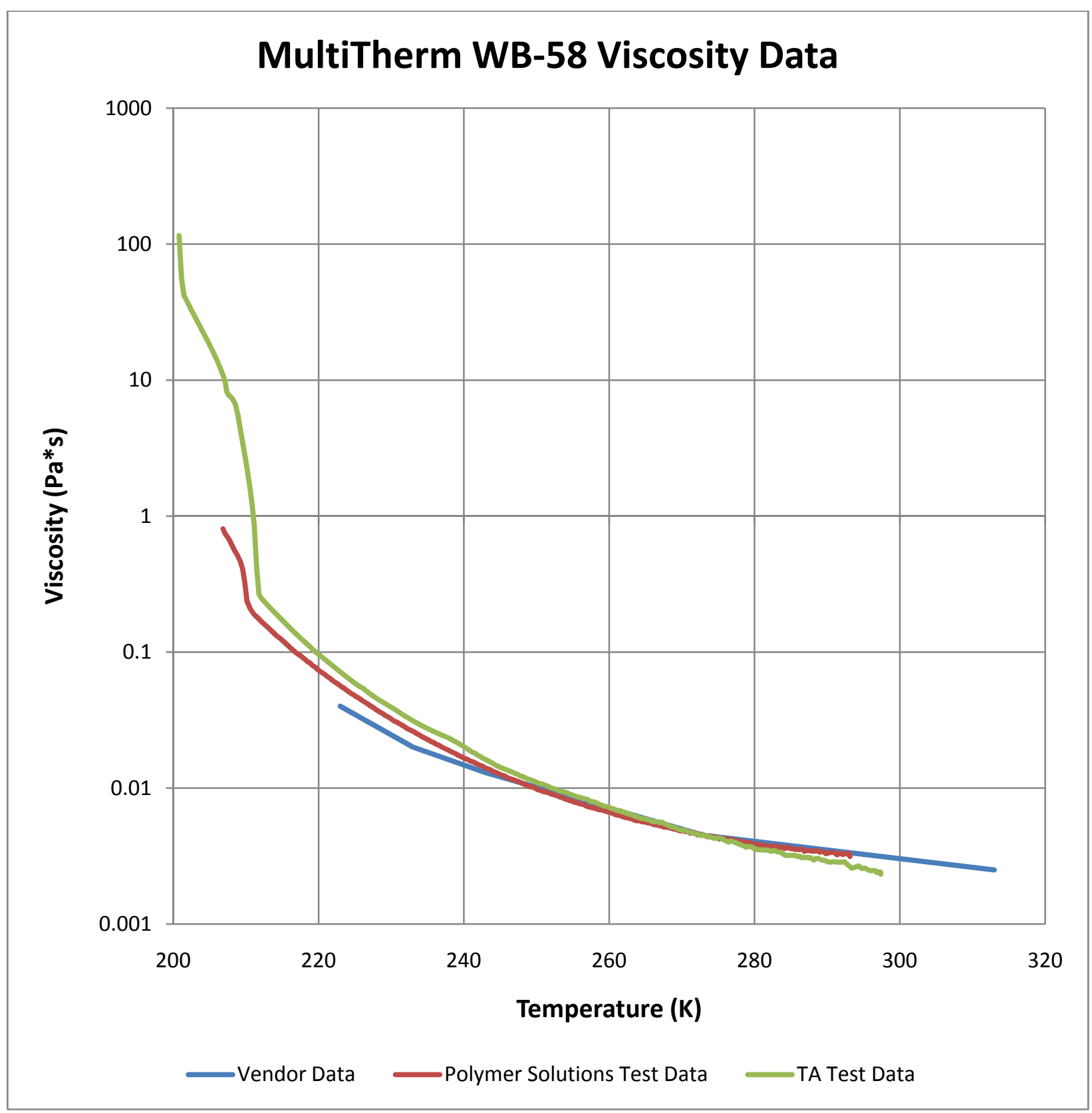

Figure 17: MultiTherm WB-58 viscosity test profiles 